\title{
PEAK1, acting as a tumor promoter in colorectal cancer, is regulated by the EGFR/ KRas signaling axis and miR-181d
}

Lanlan Huang ${ }^{1,2}$, Chuangyu Wen ${ }^{1,2}$, Xiangling Yang ${ }^{1,2}$, Qiong Lou ${ }^{1,2,3,4}$, Xiaoyan Wang ${ }^{1,2}$, Jia Che ${ }^{1,2,3,4}$, Junxiong Chen ${ }^{1,2}$, Zihuan Yang ${ }^{1,2}$, Xiaojian Wu ${ }^{1,2}$, Meijin Huang ${ }^{1,2}$, Ping Lan ${ }^{1,2}$, Lei Wang ${ }^{1,2}$, Aikichi Iwamoto ${ }^{5}$, Jianping Wang ${ }^{1,2}$ and Huanliang Liu' $1,2,3,4$

\begin{abstract}
PEAK1 is upregulated in multiple human malignancies and has been associated with tumor invasion and metastasis, but little is known about the role of PEAK1 in colorectal cancer (CRC) progression. We investigated the expression pattern, function and regulatory mechanisms of PEAK1 in CRC. Here, we found that PEAK1 is overexpressed in CRC tissues and that high PEAK1 expression predicts poor survival in colon cancer but not rectal cancer. Functionally, silencing PEAK1 inhibits cell proliferation, migration, and invasion in vitro and inhibits the growth of tumor xenografts in nude mice. Mechanistic studies revealed that PEAK1 is induced by epidermal growth factor receptor (EGFR) signaling and that PEAK1 is required for KRas-induced CRC cell growth and metastasis. Furthermore, we demonstrated that miR-181d directly targets PEAK1. Ectopic expression of miR-181d reduces the expression of PEAK1 and inhibits the growth and metastasis of CRC cells in vitro. Clinically, miR-181d is downregulated in CRC samples, and low miR-181d is correlated with poor patient survival. Our study demonstrates the importance of PEAK1 in CRC progression and suggests a potential mechanism by which increasing PEAK1 expression in CRC might be the result of EGFR/KRas signal activation and consequent miR-181d repression.
\end{abstract}

\section{Introduction}

Colorectal cancer (CRC) is the most common malignant tumor worldwide ${ }^{1}$. Distant metastasis is a major cause of death in CRC patients ${ }^{2,3}$. Currently, the standard firstline treatments that have shown promising results for metastatic colorectal cancer (mCRC) are cytotoxic chemotherapy and/or targeted therapies ${ }^{4}$. The most commonly used target therapies for $\mathrm{mCRC}$ are the monoclonal antibodies cetuximab and panitumumab,

\footnotetext{
Correspondence: Jianping Wang (wangjply@yahoo.com) or Huanliang Liu (liuhuanl@mail.sysu.edu.cn)

'Guangdong Institute of Gastroenterology, The Sixth Affiliated Hospital, Sun Yat-sen University, Guangzhou, China

${ }^{2}$ Guangdong Provincial Key Laboratory of Colorectal and Pelvic Floor Diseases, The Sixth Affiliated Hospital, Sun Yat-sen University, Guangzhou, China
} Full list of author information is available at the end of the article

These authors contributed equally: Lanlan Huang, Chuangyu Wen and Xiangling Yang.

Edited by I Amelio both of which can inactivate the EGFR signaling pathway ${ }^{5}$. Unfortunately, primary and secondary resistance after anti-EGFR antibody therapy has emerged. Recent studies have identified mutations in downstream effectors of the EGFR signaling pathway, such as $\mathrm{KRas}^{6-8}$ and other genes $^{9-11}$, as the primary cause of resistance. Therapeutic resistance to EGFR blockade could be overcome through combinatorial therapies targeting EGFR downstream genes ${ }^{9}$. Therefore, defining these genes can help guide treatment and improve clinical care.

Pseudopodium-enriched atypical kinase 1 (PEAK1), a non-receptor atypical tyrosine kinase family member KIAA2002 (sgk269), localizes to the actin cytoskeleton and focal adhesions (FAs) and regulates FA turnover ${ }^{12,13}$. PEAK1 contains multiple tyrosine phosphorylation sites $^{12}$, and one of these, Y1188, can be phosphorylated by 
EGF signaling. Such phosphorylation results in PEAK1 binding to endogenous $\mathrm{SHC} 1$ and mediating EGFR signal output ${ }^{14}$. Studies show that PEAK1 is overexpressed in multiple human malignancies and has an effect on regulating tumor migration and proliferation ${ }^{12,}{ }^{15-17}$. In summary, PEAK1 is critical for tumor development and is possibly a new therapeutic target for cancer. However, the role of PEAK1 in CRC progression and its regulatory mechanisms remain unclear. In this study, we focused on the mechanism of PEAK1 over-expression during CRC tumorigenesis.

Here, we show that PEAK1 is significantly upregulated in both colon and rectal cancer, and high PEAK1 expression predicts poor survival in colon cancer. Our study further demonstrates the importance of PEAK1 in CRC progression and indicates the activation of the EGFR/KRas signaling axis and repression of miR-181d as a potential mechanism for increased PEAK1 expression in CRC.

\section{Results}

PEAK1 overexpression is associated with poor prognosis in CRC patients

Recent studies have found that PEAK1 is overexpressed in multiple human malignancies and localizes to the actin cytoskeleton and focal adhesions ${ }^{12,15}$. To better understand the expression and localization of PEAK1 protein in $\mathrm{CRC}$, immunohistochemistry staining was performed in colon and rectal carcinoma tissue microarray (TMA) slides containing tumor tissues and adjacent normal tissues. We found that PEAK1 localized to the cytoplasm, membrane and nucleus (Fig.1a). The positive staining rate was higher in tumor tissues than in normal tissues (Fig.1b), and there was strong immunostaining in tumor tissues (Fig.1a, Supplementary Figure S1a). Significantly higher expression of PEAK1 was observed in tumors compared to adjacent normal tissues (Fig.1c, d). In addition, we found that PEAK1 was markedly upregulated in patients with lymph node metastasis compared to patients without (Fig.1e, f). We then analyzed the correlation of PEAK1 expression with clinicopathological parameters. High PEAK1 expression was significantly associated with advanced clinical stage (Table1). Kaplan-Meier analysis showed that high levels of PEAK1 expression were correlated with poor overall survival in colon cancer (Fig.1g). Further, multivariate Cox regression analysis revealed that PEAK1 expression was an independent prognostic factor for poor survival (Table2). However, PEAK1 expression was not associated with overall survival in rectal cancer (Supplementary Figure S1b, Table S1).

\section{Downregulation of PEAK1 inhibits CRC cell invasion, migration and proliferation}

PEAK1 was found to be upregulated in CRC and associated with metastasis in breast and pancreatic cancer ${ }^{12}$,
15, 16. To investigate the impact of PEAK1 on the biological properties of CRC cells, HCT 116 and HT-29 cells were depleted of PEAK1 using siRNA and tested for their ability to invade, migrate and grow in vitro. Transfection of HCT 116 and HT-29 cells with siRNA to decrease PEAK1 protein expression (Supplementary Figure S2a) markedly reduced invasion ability compared with NC cells (Fig.2a). Downregulation of PEAK1 also significantly inhibited CRC cell migration and proliferation, as determined by real-time cell migration and proliferation assays (Fig.2b, c). To investigate the role of PEAK1 in tumor growth in vivo, we used pLenti-shPEAK1 to stably knockdown endogenous PEAK1 expression in CRC cell. Transfection of CRC cells with pLenti-shPEAK1 caused decreased PEAK1 protein expression and consequently reduced cell invasion compared with control cells in vitro (Supplementary Figure S2b and c). HCT 116 cells stably transfected with pLenti-shPEAK1 or pLenti-vector were subcutaneously injected into the flanks of nude mice $(n=$ 12). HCT 116-shPEAK1-formed tumors showed decreased volume and weight compared with pLentivector tumors (Fig.2d, e). To further characterize the role of PEAK1 in CRC, we performed a gene-expression microarray assay in HCT 116 cells following siRNAmediated PEAK1 knockdown. We found that 622 genes were upregulated and 744 were downregulated at least two-fold in HCT 116-siRNA cells (Data set S1). KEGG pathway analysis revealed that the MAPK, Focal adhesions and the PI3K-Akt signaling pathway were the most downregulated pathways in cells transfected with siRNA (Supplementary Figure S3).

\section{EGFR signaling increases the expression of PEAK1}

EGF and other EGF-like ligands trigger EGFR, which activates downstream pro-oncogenic signaling pathways, including the MAPK cascade (RAS-RAF-MEK-ERK) and PI3K-AKT-mTOR pathway, regulating cancer cell survival, growth and motility ${ }^{18}$. As previous studies have suggested, PEAK1 takes part in regulating growth factor receptor signal output ${ }^{14}$. To ascertain this relationship, we first evaluated the expression correlations between EGFR and PEAK1 using public data from The Cancer Genome Atlas (TCGA). Spearman's correlation analyses showed that EGFR significantly and positively correlated with PEAK1 levels in CRC patients $(n=465$, Fig.3a). Then, we investigated the effect of EGF stimulation on PEAK1 expression in CRC. HCT 116 and $\mathrm{CaCO}_{2}$ were treated with EGF for $1 \mathrm{~h}$ and subjected to western blot. The results showed that EGF treatment trigger EGFR/Erk signaling by increased the levels of p-EGFR and p-Erk $1 / 2$. PEAK1 expression could be stimulated by EGF in a dosedependent manner (Fig.3b). CRC cells were treated with EGF followed by siPEAK1 transfection. The cells were harvested after $1 \mathrm{~h}$ of EGF treatment. As expected, siRNA- 


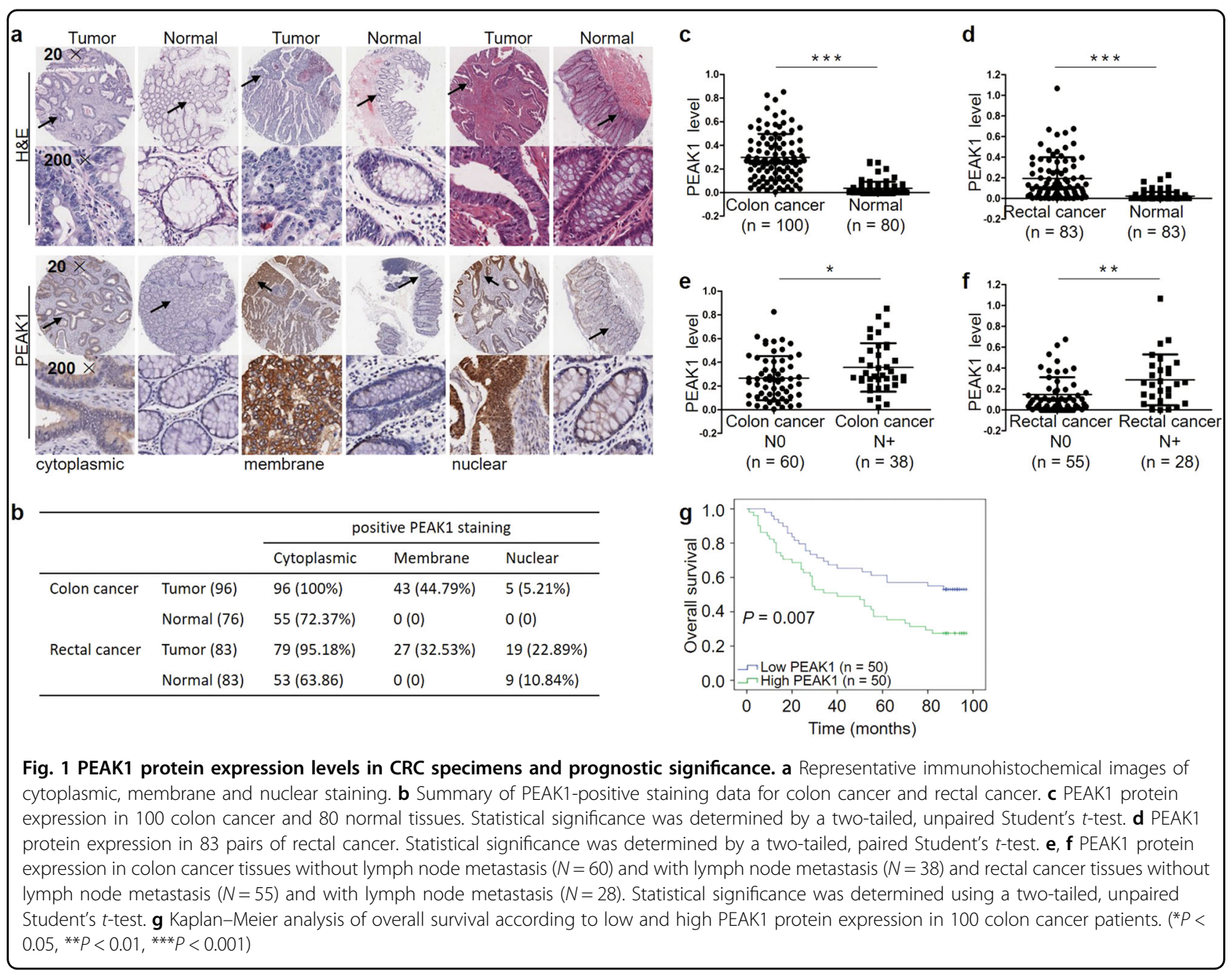

PEAK1 significantly attenuated EGF-induced p-Erk1/2 levels (Fig.3c). Taken together, these results indicate that PEAK1 expression is regulated by EGFR signaling in CRC cells.

\section{Downregulation of PEAK1 inhibits EGFR/KRas signaling}

Over-activation of the EGFR cascade, by KRas gene mutation for example, promotes cell growth, proliferation, migration, and inhibition of apoptosis ${ }^{10,}{ }^{19-22}$. Our studies have demonstrated that silencing PEAK1 decreased the activity of the EGFR/Erk signaling pathway (Fig.3c, Supplementary Figure S3). Hence, to explore whether KRas regulates PEAK1 expression in CRC, we evaluated the correlation of the expression of KRas and PEAK1. Data from TCGA showed that PEAK1 significantly and positively correlated with KRas levels in CRC patients ( $n=465$, Fig.4a). To test the effects of KRas on endogenous levels of PEAK1 in CRC cells, we used qRT-PCR and western blot assays to measure the mRNA and protein levels of PEAK1 in HCT 116 and $\mathrm{CaCO}_{2}$ cells infected with a pLenti- KRas or siRNA-KRas.
As shown in Fig.4b,c, overexpression of KRas by pLentiKRas significantly increased PEAK1 mRNA and protein expression and the levels of p-Erk1/2. Downregulation of KRas by siRNA significantly decreased PEAK1 mRNA and protein levels and the levels of p-Erk1/2 (Fig.4d, e). Taken together, these results demonstrate that KRas induces PEAK1 expression in CRC.

\section{Downregulation of PEAK1 impairs KRas-induced growth and metastasis in vitro}

The above results strongly suggested that PEAK1 expression might be necessary for the KRas-induced biological properties of CRC cells. Therefore, we investigated whether knocking down PEAK1 expression could impair motility and growth in KRas-overexpressing cells. Western blot showed that transfection with siRNAPEAK1 significantly attenuated KRas-upregulated PEAK1 levels (Fig.5a). Then, we tested the effect of PEAK1 knockdown on cell motility by Wound-healing assay and Matrigel invasion assay. As shown in Fig.5b, c, transfection with pLenti-KRas increased cell migration 
Table 1 Correlation between PEAK1 expression and clinical parameters in colorectal cancer patients

\begin{tabular}{|c|c|c|c|c|c|c|c|c|}
\hline \multirow[t]{2}{*}{ Factors } & \multicolumn{4}{|c|}{ Colon cancer } & \multicolumn{4}{|c|}{ Rectal cancer } \\
\hline & $n$ & Low PEAK1 (\%) & High PEAK1(\%) & $P$ value & $n$ & Low PEAK1 (\%) & High PEAK1(\%) & $P$ value \\
\hline Age & & & & 0.149 & & & & 0.934 \\
\hline$<60$ & 20 & $12(60 \%)$ & $8(40 \%)$ & & 30 & 15 (50.0\%) & $15(50.0 \%)$ & \\
\hline$\geq 60$ & 74 & 31 (41.9\%) & $43(58.1 \%)$ & & 53 & 27 (50.9\%) & $26(49.1 \%)$ & \\
\hline Gender & & & & 0.753 & & & & 0.309 \\
\hline Female & 44 & $21(47.7 \%)$ & $23(52.3 \%)$ & & 35 & $20(57.1 \%)$ & 15 (42.9\%) & \\
\hline Male & 55 & $28(50.9 \%)$ & 27 (49.1\%) & & 48 & 22 (45.8\%) & $26(54.2 \%)$ & \\
\hline TNM stage & & & & $0.02^{*}$ & & & & $0.001^{*}$ \\
\hline $1+\|$ & 60 & 35 (58.3\%) & $25(41.7 \%)$ & & 55 & 35 (63.6\%) & $20(36.4 \%)$ & \\
\hline III+IV & 38 & 13 (34.2\%) & $25(65.8 \%)$ & & 28 & 7 (25.0\%) & $21(75.0 \%)$ & \\
\hline$p T$ & & & & $0.05^{*}$ & & & & 0.054 \\
\hline $\mathrm{T} 1+\mathrm{T} 2$ & 7 & $6(85.7 \%)$ & $1(14.3 \%)$ & & 22 & 15 (68.2\%) & 7 (31.8\%) & \\
\hline $\mathrm{T} 3+\mathrm{T} 4$ & 89 & $42(47.2 \%)$ & $47(52.8 \%)$ & & 61 & $27(44.3 \%)$ & $34(55.7 \%)$ & \\
\hline$p N$ & & & & $0.02^{*}$ & & & & $0.001^{*}$ \\
\hline NO & 60 & 35 (58.3\%) & $25(41.7 \%)$ & & 55 & 35 (63.6\%) & $20(36.4 \%)$ & \\
\hline $\mathrm{N} 1+\mathrm{N} 2$ & 38 & 13 (34.2\%) & $25(65.8 \%)$ & & 28 & 7 (25.0\%) & $21(75.0 \%)$ & \\
\hline$p M$ & & & & 0.582 & & & & 0.309 \\
\hline MO & 97 & $48(49.5 \%)$ & 49 (50.5\%) & & 82 & $42(51.2 \%)$ & 40 (48.8\%) & \\
\hline M1 & 3 & 1 (33.3\%) & $2(66.7 \%)$ & & 1 & $0(0.0 \%)$ & $1(100 \%)$ & \\
\hline
\end{tabular}

Table 2 Univariate and multivariate analyses of various potential prognostic factors in 100 colon cancer patients

\begin{tabular}{|c|c|c|c|c|}
\hline \multirow[t]{2}{*}{ Factors } & \multicolumn{2}{|l|}{ Univariate analysis } & \multicolumn{2}{|l|}{ Multivariate analysis } \\
\hline & HR $(95 \% \mathrm{Cl})$ & $P$ & HR $(95 \% \mathrm{Cl})$ & $P$ \\
\hline Age & $1.012(0.988,1.036)$ & 0.337 & & \\
\hline Gender & $0.987(0.590,1.650)$ & 0.959 & & \\
\hline TNM Stage & $2.565(1.625,4.049)$ & $<0.001^{*}$ & $2.365(1.454,3.846)$ & $0.001^{*}$ \\
\hline PEAK1 expression & $6.259(1.718,22.808)$ & $0.005^{*}$ & $5.724(1.430,22.912)$ & $0.014^{*}$ \\
\hline
\end{tabular}

${ }^{*} P<0.05 ;$ HRhazard ratio, Clconfidence interval.

and invasion, whereas knocking down PEAK1 significantly reduced KRas-induced cell migration and invasion. Next, we tested the effect of PEAK1 knockdown on KRas-induced growth. As shown in Fig.5d, e, KRas promoted colony formation and proliferation. In contrast, downregulation of PEAK1 attenuated KRasinduced growth (Fig.5d, e). Taken together, these findings show that PEAK1 is necessary for KRas-induced invasion, migration, colony formation and proliferation in CRC cells.
PEAK1 is a direct target of miR-181d in CRC cells

Linear regression analysis revealed that EGFR $\left(r^{2}=\right.$ $0.163)$ and KRas $\left(r^{2}=0.063\right)$ do not completely explain the expression pattern of PEAK1 in clinical CRC specimens. Here, we investigated whether miRNAs regulate levels of PEAK1 in CRC cells. To find miRNAs associated with the regulation of PEAK1 expression, a bioinformatics search was performed for potential miRNAs targeting PEAK1 mRNA using common databases such as miRWalk and TargetScan. The results showed that miR-181d 


\section{a}

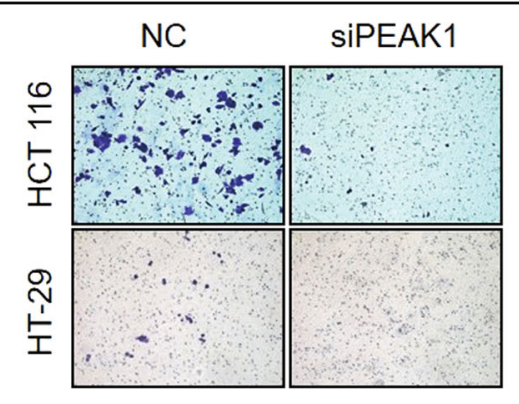

b

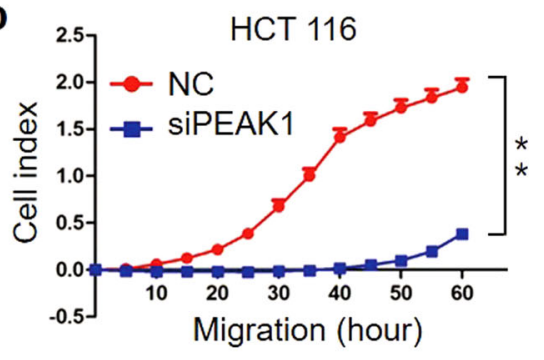

C

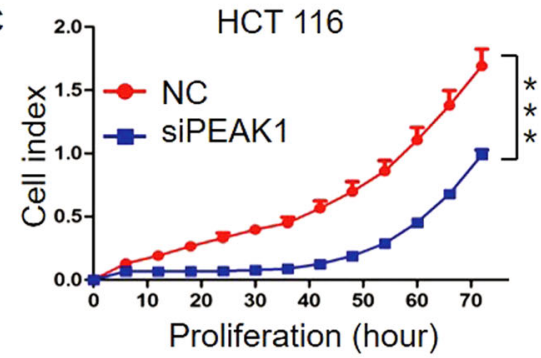

d

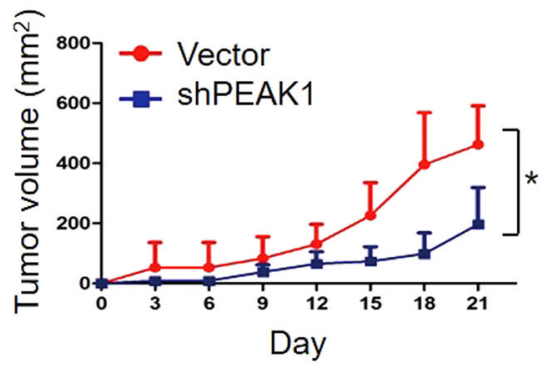

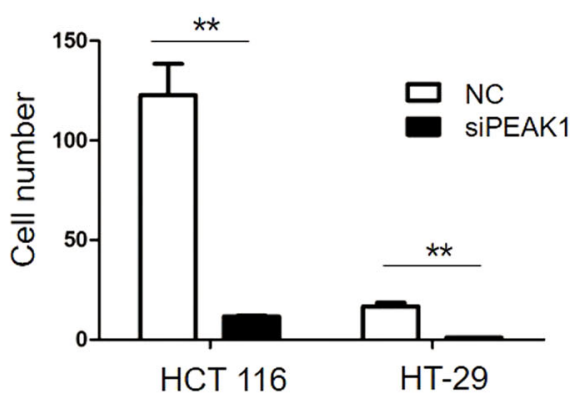
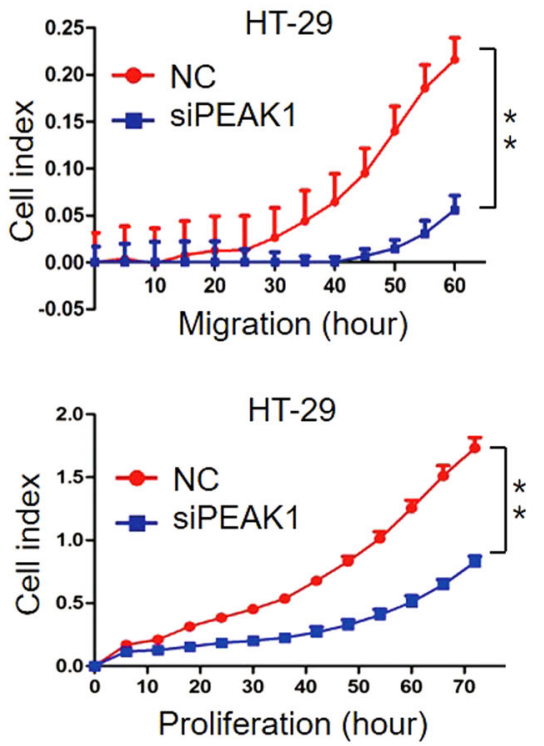

e
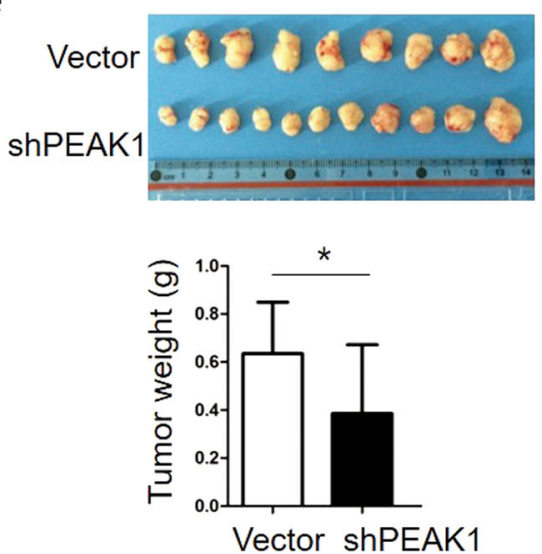

Fig. 2 Downregulation of PEAK1 inhibits CRC cell invasion, migration and proliferation. a Transwell assays were used to estimate the effects of PEAK1 downregulation on CRC cell invasion abilities. $\mathbf{b}$, $\mathbf{c}$ Real-time migration and real-time proliferation of CRC cells transfected with NC or siPEAK1. The delta cell index indicates electrical impedance measurements. All the above experiments were carried out in triplicate. $\mathbf{d}$ The tumor growth curve. HCT 116 cells were transfected with pLenti-shRNA or pLenti-vector and subcutaneously injected into nude mice. Statistical significance was determined by Student's paired $t$-test. ( $n=12$ per group). e Photographs of the tumors at day 21 after inoculation with HCT 116 cells transfected with pLenti-shRNA or pLenti-vector (up). Average tumor weight after tumor excision (down). (Data are represented as the mean \pm s.d. ${ }^{*} P<0.05,{ }^{* *} P<0.01$ )

was a potential miRNA targeting PEAK1 because miR181d incompletely complemented the $3^{\prime} \mathrm{UTR}$ region of PEAK1 (Fig.6a). To verify whether PEAK1 was a direct target of miR-181d, we cloned the $3^{\prime} \mathrm{UTR}$ region of PEAK1 mRNA, which included the predicted miR-181d recognition site, and then inserted it into a luciferase 

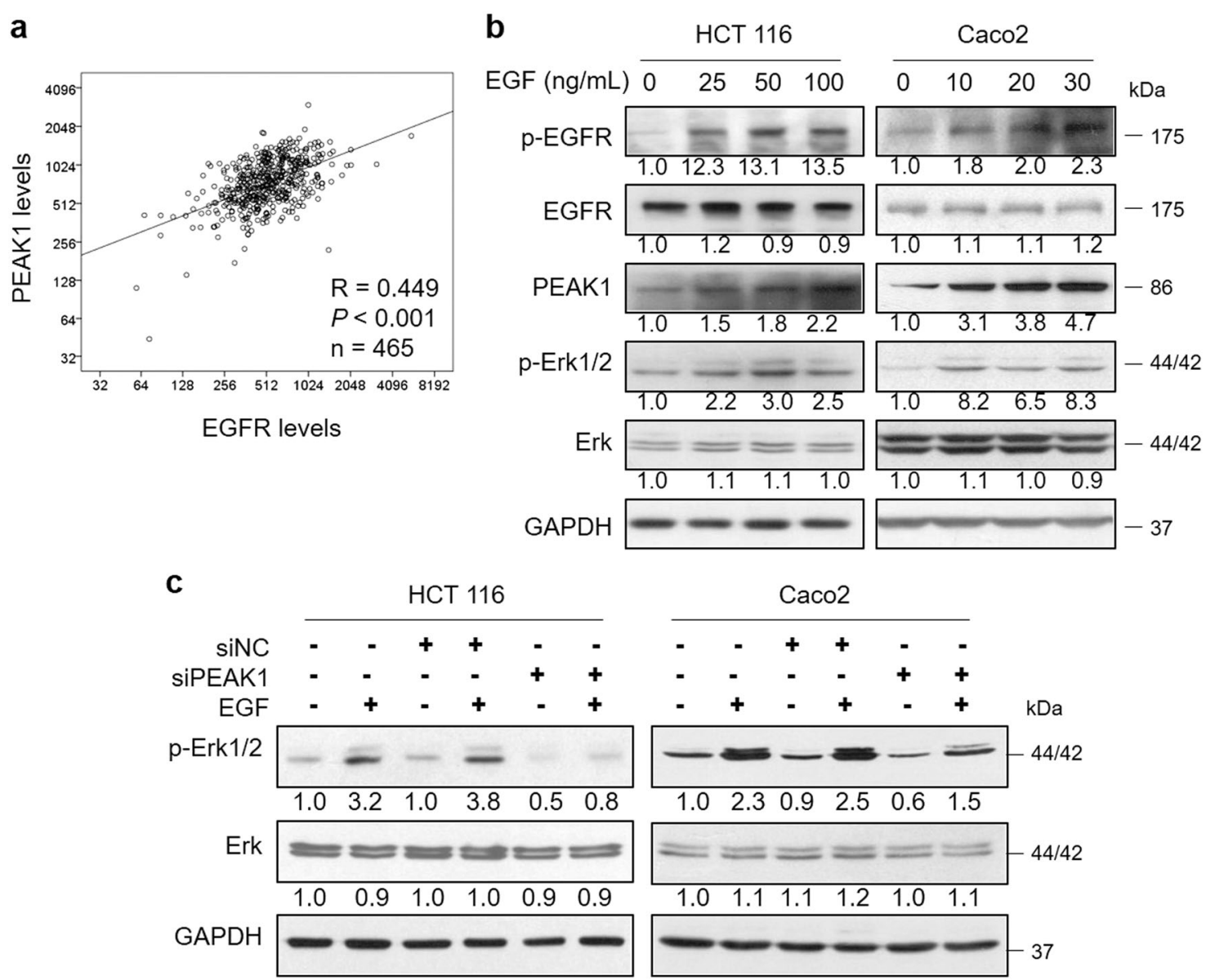

Fig. 3 EGFR signaling increases the expression of PEAK1. a The correlation between EGFR and PEAK1 expression was evaluated by Spearman's method. $\mathbf{b}$ Western blot analyses of EGFR, PEAK1 and Erk in total extracts from CRC cells treated with EGF for $1 \mathrm{~h}$. c CRC cells were treated with EGF, followed by siPEAK1 transfection, and then western blot was performed to analyze the Erk levels. These experiments were repeated three times

reporter plasmid. The miR-181d binding site in the $3^{\prime} \mathrm{UTR}$ region of PEAK1 was mutated to obtain the $3^{\prime} \mathrm{UTR}$ MutPEAK1-luc plasmid (Fig.6a). Transient transfection of wild-type PEAK1-luc reporter with miR-181d mimics into HCT 116 cells led to a significant decrease in luciferase activity compared to the activity of NC (Fig.6b). However, the decrease in luciferase activity of Mutant MutPEAK1luc compared to that in the NC group was not significant (Fig.6b). To determine whether miR-181d affects PEAK1 expression in the intracellular environment in CRC, the expression of PEAK1 was evaluated in HCT 116 and $\mathrm{CaCO}_{2}$ cells following transfection with either miR-181d mimics or inhibitors (anti-miR-181d). The transfection efficiency was confirmed by qRT-PCR (Supplementary Figure S4a). Transfection with miR-181d mimics resulted in a significant reduction of PEAK1 protein expression (Fig.6c). Downregulation of miR-181d using anti-miR181d was associated with significantly higher expression of PEAK1 protein (Fig.6c). We also investigated the correlation between the expression levels of miR-181d and the EGFR/Erk signaling pathway. As expected, miR-181d over-expression led to a significant decrease in PEAK1 protein levels and in the levels of p-Erk1/2 (Fig.6d). The results make it evident that miR-181d affects PEAK1 expression by directly binding to the $3^{\prime} \mathrm{UTR}$ region of PEAK1 and validating that PEAK1 is a direct target of miR-181d.

\section{Ectopic expression of miR-181d decreases the invasive,} migratory and proliferative capacities of CRC cells in vitro

Our studies demonstrated that miR-181d targets PEAK1. Therefore, we next investigated the role of miR181d in CRC cells. HCT 116 cells were stably infected with the pLenti-miR-181d or pLenti-vector (Supplementary Figure S4b). Overexpressing miR-181d resulted in a reduction in PEAK1 expression (Supplementary Figure S4c). Invasion assays showed that ectopic miR-181d expression significantly decreased the invasive ability of HCT 116 cells (Fig.6e). However, transfection of CRC cells with the miR-181d inhibitor enhanced cell invasion 


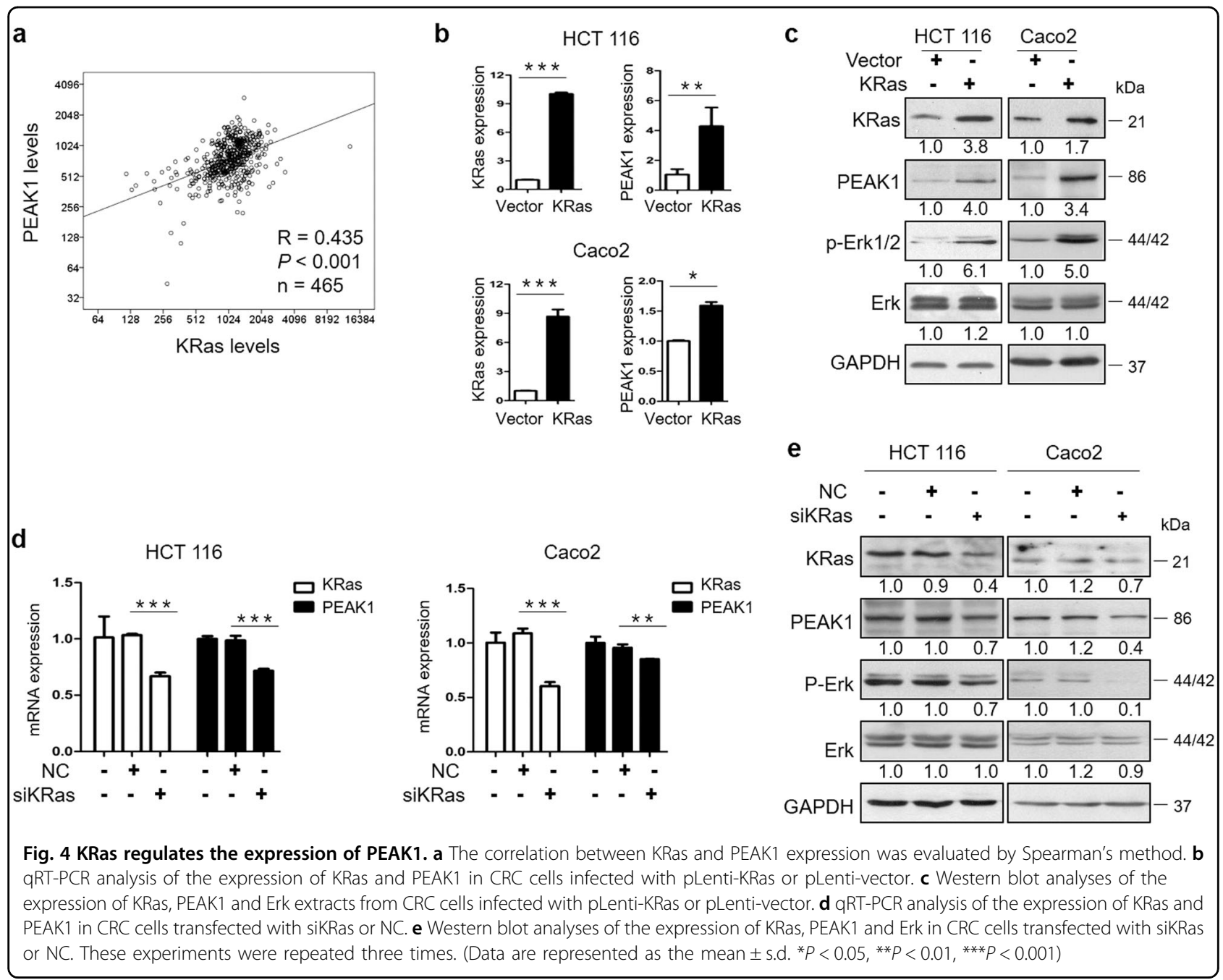

compared with NC cells (Fig.6e). Real-time cell migration assays showed a reduced migration of miR-181doverexpressing cells (Fig.6f). Colony formation and realtime cell proliferation assays showed that ectopic miR181d expression decreased colony formation and cell proliferation (Fig.6g, h). Taken together, these findings indicate that miR-181d acts as a tumor suppressor in CRC.

\section{miR-181d is an independent prognostic factor for CRC}

Our studies have shown that high PEAK1 expression is associated with poor overall survival in colon cancer. To further investigate the clinical pathology and prognostic significance of miR-181d expression, in situ hybridization (ISH) staining for miR-181d was performed on 353 CRC samples in a tissue microarray. The positive staining of tissue was expressed as blue-violet (Fig.6i). As shown in Supplementary Table S2, miR-181d expression was significantly correlated with tumor location, local relapse and TNM stage. The Kaplan-Meier curve and log-rank test showed that downregulation of miR-181d was significantly associated with poorer overall survival in CRC (Fig.6j). In multivariate analysis using the Cox proportional hazards model, miR-181d expression was found to be an independent prognostic factor for CRC (Supplementary Table S3).

\section{Discussion}

In this study, we found that PEAK1 was a prognosisassociated marker that was upregulated in CRC. Our immunohistochemistry results showed that PEAK1 localized to the cytoplasm, membrane and nucleus. These findings are consistent with the results of the work of Wang et al. ${ }^{12}$, who reported that PEAK1 localized to the actin cytoskeleton and FAs in migrating cells. We also found that PEAK1 was exclusively localized to the membrane of CRC cells, indicating that spatiotemporal regulation of PEAK1 was disrupted in tumor tissues. In addition, we showed that PEAK1 protein expression was increased in CRC tissues in comparison with normal 


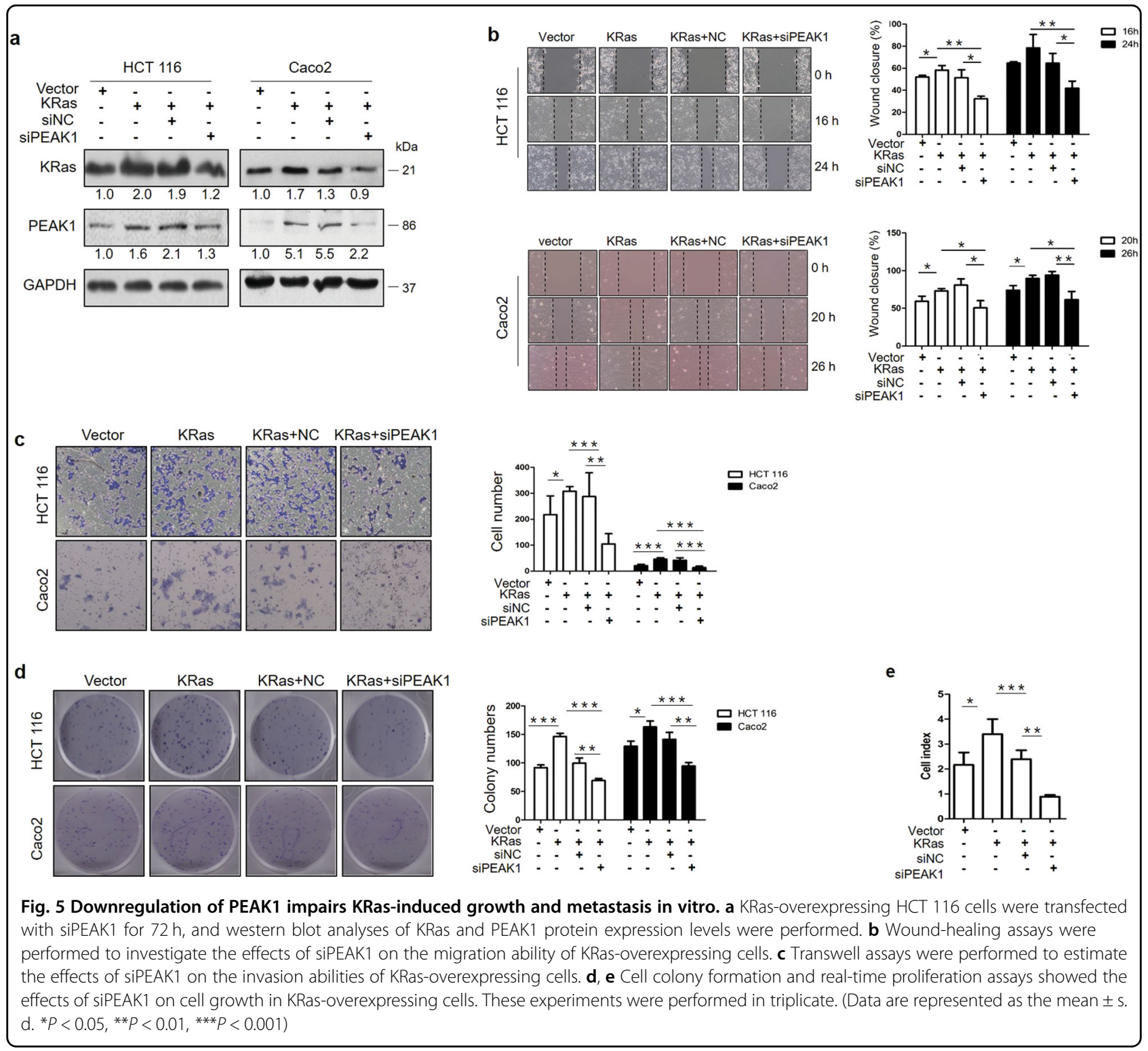

tissues and correlated with TNM stage. Subsequent analyses involving 264 CRC patients from the TCGA llluminaGA_RNASeqV2 data set confirmed that high PEAK1 expression was associated with poor overall survival in CRC (Supplementary Figure S1c, Table S4 and 5). In two cohort studies of CRC, high levels of PEAK1 expression were associated with poorer overall survival, indicating that PEAK1 has a critical role in CRC development.

Previous studies have identified PEAK1 as a positive regulator of cell growth and metastasis in breast cancer and pancreatic cancer ${ }^{15}, 17,23$. However, the role of PEAK1 in CRC remains unknown. Here, we found that downregulation of PEAK1 inhibited CRC cell invasion, migration, and proliferation. We then investigated possible pathways by which PEAK1 could be involved in CRC.
Our studies showed that down-regulating PEAK1 inactivated EGFR signaling (MAPK and PI3K-Akt signaling pathway) and the focal adhesion signaling pathway (Supplementary Figure S3). Recently, numbers of studies have shown roles for activated MAPK and PI3K-Akt signaling in the regulation of metastasis and proliferation in cancer ${ }^{24-29}$. The above studies suggest that downregulation of PEAK1 expression decreases EGFR signaling, thereby decreasing CRC cell invasion, migration, and proliferation.

Previous studies have shown that PEAK1 takes part in the EGFR signal output ${ }^{14}$. PEAK1 protein expression is positively regulated by KRas/Src and KRas/eIF5A signaling in pancreatic cancer ${ }^{15}, 30,31$. Our gene-expression microarray assay results showed that downregulation of 




PEAK1 inactivated a number of pathways, including the MAPK and PI3K-Akt signaling pathways, indicating that PEAK1 may be involved in EGFR signaling transduction. Our studies also showed that PEAK1 expression was significantly positively correlated with EGFR and KRas levels in CRC patients. EGF stimulation showed that EGFR induced PEAK1 expression, while inhibiting the expression of PEAK1 impaired EGF/Erk signaling stimulated by EGF. Furthermore, we found that KRas could induce PEAK1 expression and that PEAK1 was necessary for KRas-induced growth and metastasis in CRC. Together, these results demonstrate that PEAK1 is under the regulation of the EGFR/KRas signaling axis and promotes an aggressive phenotype in CRC.

Multiple linear regression analysis revealed that EGFR and KRas did not completely explain the expression pattern of PEAK1 in clinical CRC specimens. In fact, our statistical analysis using the TCGA data set showed elevated PEAK1 mRNA expression in normal tissue compared with cancer tissue (Supplementary Figure S1d). Possible explanations for these apparently opposite results include differences in mRNA and protein levels of PEAK1. For example, in breast cancer, the PEAK1 expression pattern was not reflected in the relative mRNA levels, indicating that elevated PEAK1 expression in breast cancer cells must be mediated via a post-transcriptional or post-translational mechanism ${ }^{16}$. MicroRNAs (miRNAs) are small (19-25 nt), noncoding, regulatory RNAs that regulate gene expression by complementary base pairing with the $3^{\prime}$ untranslated region (UTR) of target messenger RNAs (mRNAs), causing mRNA degradation or suppressing mRNA translation ${ }^{32,33}$. Up to $30 \%$ of 
human genes appear to be conserved miRNA targets ${ }^{33}$. miRNAs were reported to be associated with pathogenesis and could be used as diagnostic and prognostic biomarkers in many human cancers ${ }^{34-38}$. miRNAs are known to regulate gene expression at the post-transcriptional level ${ }^{32,} 39$. Our study investigated the potential involvement of a miRNA-mediated mechanism in the increased expression of PEAK1 in CRC. We performed a bioinformatics search for potential miRNAs targeting PEAK1 mRNA and found that miR-181d had the highest predictive scores, indicating that miR-181d might directly target PEAK1. The Luciferase activity assay performed later confirmed our suspicion. Together, these findings suggest that low expression of miR-181d leads to high expression of PEAK1 in CRC.

It has been reported that miR-181d is downregulated in glioma and acts as a tumor suppressor by targeting KRas and Bcl- $-2^{40}$. High expression of miR-181d was associated with improved overall survival in glioblastoma ${ }^{41,} 42$. Exogenous over-expression of miR-181d inhibited the proliferation of pancreatic cancer cells ${ }^{43}$. Guo et al. ${ }^{44}$ reported that miR-181d functions as a tumor promoter in CRC. To better understand the role of miR-181d in CRC, the clinical significance and biological function of miR$181 \mathrm{~d}$ were analyzed. Our results showed that miR-181d expression was significantly correlated with local relapse and TNM stage, and the downregulation of miR-181d was significantly associated with poorer overall survival in CRC. In addition, gain-of-function and loss-of-function assays were performed to assess the effect of miR-181d on CRC invasion and metastasis. The results showed that silencing miR-181d upregulated PEAK1 and strengthened cell invasion in vitro, whereas overexpressing miR-181d inhibited PEAK1 expression as well as cell proliferation, invasion and migration in vitro. Hence, miR-181d is an important tumor suppressor miRNA in CRC invasion and metastasis, and PEAK1 is downstream effector of miR$181 \mathrm{~d}$ in its target network. To conclude, we show a novel regulatory mechanism of PEAK1 expression in CRC in which miR-181d suppresses its direct target, PEAK1, in turn regulating CRC invasion and metastasis.

Together, our data demonstrate an association between PEAK1 expression and worse prognosis in CRC and the mechanism of PEAK1 over-expression during CRC tumorigenesis.

\section{Materials and methods \\ Human samples}

CRC TMA slides used for immunohistochemistry analysis of PEAK1 protein expression were purchased from Shanghai Outdo Biotech (Shanghai, China). CRC TMA slides used for ISH analysis of miR-181d expression were obtained from the tumor bank of the Department of Pathology of the First Affiliated Hospital, Sun Yat-sen
University (Guangzhou, China). The procedure for human sample collection was approved by the Ethical Committee of Sun Yat-sen University (Guangzhou, China), and written informed consent was obtained from all of the patients. All data were analyzed anonymously, and all experiments were in compliance with the Helsinki Declaration.

\section{Immunohistochemistry of PEAK1}

Immunohistochemistry was performed using a PEAK1 antibody (Sigma-Aldrich, St. Louis, MO, USA). The detailed procedures are described in the Supplemental materials and methods.

\section{Western blot analysis}

Cell lysates ( $40 \mu$ g protein/line) were separated on a $10 \%$ SDS-PAGE gel and transferred to a polyvinylidene fluoride (PVDF) membrane (Millipore, Billerica, MA, USA). The blotted membranes were blocked with 5\% skim milk or $5 \%$ bovine serum albumin and incubated overnight at $4{ }^{\circ} \mathrm{C}$. Anti-PEAK1 (86 kDa, Abnova, Taipei, Taiwan), antiEGFR (Tyr1173, $175 \mathrm{kDa})$, anti-EGFR $(175 \mathrm{kDa})$, antiKRas (21 kDa), anti-phospho-p44/42 Erk (Thr202/ Tyr204, 44/42 kDa) anti-p44/42 Erk (44/42 kDa) (Cell Signaling Technology, Danvers, MA, USA), anti- $\beta$-actin $(43 \mathrm{kDa})$ and anti-GAPDH (36 kDa) (Abcam, Cambridge, UK) antibodies were used. The detailed procedures are described in the Supplemental materials and methods.

\section{In situ hybridization of miR-181d}

ISH was performed as described previously ${ }^{45}$. Briefly, ISH was performed using a hsa-miR-181d probe from Exiqon (miRCURY LNA Detection probe, $250 \mathrm{pmol}$, 5'DIG and 3 -DIG labeled). Detection of the probe was carried out using anti-digoxigenin-AP (Roche, Germany), and the hybridized probes were detected by applying a BCIP/NBT Alkaline Phosphatase Color Development Kit. No-probe controls were included for each hybridization procedure. Images were taken using a Leica DMI 4000B inverted microscope (Leica Micro-systems, Wetzlar, Germany). ISH staining of the image was analyzed using Image-Pro-Plus 6.0 (Media Cybernetics, CA, USA).

\section{Cell proliferation, migration and invasion assays}

Cell proliferation and migration assays were performed on the xCELLigence system from ACEA Biosciences. Cell invasion assays were performed on Transwell chambers pre-coated with Matrigel (BD Bioscience, San Jose, CA, USA). Detailed procedures are described in the Supplemental Materials and Methods.

\section{Animal study}

All animal studies were conducted in accordance with German animal welfare law and approved by the 
Institutional Animal Care and Use Committee of Sun Yatsen University. HCT 116 cells transfected with pLentishRNA or pLenti-vector were harvested by trypsin, washed with PBS, and resuspended in RPMI medium supplemented with $10 \%$ FBS. A total of $5 \times 10^{6}$ cells were subcutaneously transplanted into the flanks of 5 -week-old nude mice (six mice per group). Injections were performed in both flanks of each animal. Tumor volumes were measured with calipers and calculated as length $x$ width $^{2} \times 0.4$. The tumor sizes were measured at 3-day intervals as soon as the tumors were measurable. On day 21 , the tumor masses were measured, excised, and further analyzed.

\section{Statistical analysis}

Statistical analysis was performed using SPSS18.0 software (SPSS, IBM, Chicago, IL, USA). Data are expressed as the mean \pm s.d., and statistical significance was determined with Student's $t$-tests. Statistical comparisons between groups were analyzed using Student's paired $t$-test. $P$-values $<0.05$ were considered statistically significant. Correlations between clinicopathological features and PEAK1/miR-181d expression were calculated according to the Chi-square test. The cumulative survival time was calculated utilizing the Kaplan-Meier method and analyzed with the log-rank test. Univariate and multivariate analyses were performed based on the Cox proportional hazards regression model.

\section{Acknowledgements \\ This work was supported by the National Natural Science Foundation of China (81672413 and 81402418); Guangdong Provincial Department of Science and Technology (2014B020212016); Guangdong Natural Science Funds (2015A030310096); Guangzhou Science Technology and Innovation Commission (2016201604030007; 2016201604030003); Overseas Excellent Professor Project, Ministry of Education, China; Japan Ministry of Education, Culture, Sports, Science and Technology (MEXT) for Program of Japan Initiative for Global Research Network on Infectious Diseases (J-GRID); China Postdoctoral Science Foundation (2016M592583); and National Key Clinical Discipline.}

\begin{abstract}
Author details
${ }^{1}$ Guangdong Institute of Gastroenterology, The Sixth Affiliated Hospital, Sun Yat-sen University, Guangzhou, China. ${ }^{2}$ Guangdong Provincial Key Laboratory of Colorectal and Pelvic Floor Diseases, The Sixth Affiliated Hospital, Sun Yatsen University, Guangzhou, China. ${ }^{3}$ Institute of Human Virology and Key Laboratory of Tropical Disease Control (Ministry of Education), Sun Yat-sen University, Guangzhou, China. ${ }^{4}$ Department of Clinical Laboratory, The Sixth Affiliated Hospital, Sun Yat-sen University, Guangzhou, China. ${ }^{5}$ Advanced Clinical Research Center, Institute of Medical Science, University of Tokyo, Tokyo, Japan
\end{abstract}

Conflict of interest

The authors declare that they have no conflict of interest.

\section{Publisher's note}

Springer Nature remains neutral with regard to jurisdictional claims in published maps and institutional affiliations.
Supplementary Information accompanies this paper at https://doi.org/ 10.1038/s41419-018-0320-8.

Received: 21 September 2017 Revised: 12 January 2018 Accepted: 15 January 2018

Published online: 15 February 2018

\section{References}

1. Siegel, R., Desantis, C. \& Jemal, A. Colorectal cancer statistics, 2014. CA Cancer J. Clin. 64, 104-117 (2014).

2. Rees, M., Tekkis, P. P., Welsh, F. K., O'Rourke, T. \& John, T. G. Evaluation of longterm sunvival after hepatic resection for metastatic colorectal cancer: a multifactorial model of 929 patients. Ann. Surg. 247, 125-135 (2008).

3. März, L. \& Piso, P. Treatment of peritoneal metastases from colorectal cancer. Gastroenterol. Rep. (Oxf.) 3, 298-302 (2015).

4. Prenen, H., Tejpar, S. \& Van Cutsem, E. New strategies for treatment of KRAS mutant metastatic colorectal cancer. Clin. Cancer Res 16, 2921-2926 (2010).

5. Van Cutsem, E., Cervantes, A., Nordlinger, B. \& Arnold, D., ESMO Guidelines Working Group. Metastatic colorectal cancer: ESMO clinical practice guidelines for diagnosis, treatment and follow-up. Ann. Oncol. 25(Suppl. 3), iii1-iii9 (2014).

6. Misale, S. et al. Emergence of KRAS mutations and acquired resistance to antiEGFR therapy in colorectal cancer. Nature 486, 532-536 (2012).

7. Mao, C. et al. KRAS p.G13D mutation and codon 12 mutations are not created equal in predicting clinical outcomes of cetuximab in metastatic colorectal cancer: a systematic review and meta-analysis. Cancer 119, 714-721 (2013).

8. Diaz, L. A. Jr. et al. The molecular evolution of acquired resistance to targeted EGFR blockade in colorectal cancers. Nature 486, 537-540 (2012).

9. Bertotti, A. et al. The genomic landscape of response to EGFR blockade in colorectal cancer. Nature 526, 263-267 (2015).

10. Hsu, H. C. et al. Mutations of KRAS/NRAS/BRAF predict cetuximab resistance in metastatic colorectal cancer patients. Oncotarget 7, 22257-22270 (2016).

11. De Roock, W. et al. Effects of KRAS, BRAF, NRAS, and PIK3CA mutations on the efficacy of cetuximab plus chemotherapy in chemotherapy-refractory metastatic colorectal cancer: a retrospective consortium analysis. Lancet Oncol. 11, 753-762 (2010).

12. Wang, Y. et al. Pseudopodium-enriched atypical kinase 1 regulates the cytoskeleton and cancer progression [corrected]. Proc. Natl. Acad. Sci. USA 107, 10920-10925 (2010).

13. Bristow, J. M., Reno, T. A., Jo, M., Gonias, S. L. \& Klemke, R. L. Dynamic phosphorylation of tyrosine 665 in pseudopodium-enriched atypical kinase 1 (PEAK1) is essential for the regulation of cell migration and focal adhesion turnover. J. Biol. Chem. 288, 123-131 (2013).

14. Zheng, Y. et al. Temporal regulation of EGF signalling networks by the scaffold protein Shc1. Nature 499, 166-171 (2013).

15. Kelber, J. A. et al. KRas induces a Src/PEAK1/ErbB2 kinase amplification loop that drives metastatic growth and therapy resistance in pancreatic cancer. Cancer Res 72, 2554-2564 (2012).

16. Croucher, D. R. et al. Involvement of Lyn and the atypical kinase SgK269/PEAK1 in a basal breast cancer signaling pathway. Cancer Res 73, 1969-1980 (2013).

17. Agajanian, M. et al. PEAK1 acts as a molecular switch to regulate contextdependent TGFbeta responses in breast cancer. PloS one. PLoS One 10, e0135748 (2015).

18. Leto, S. M. \& Trusolino, L. Primary and acquired resistance to EGFR-targeted therapies in colorectal cancer: impact on future treatment strategies. J. Mol. Med (Berl.) 92, 709-722 (2014).

19. Sangodkar, J. et al. Activation of tumor suppressor protein PP2A inhibits KRASdriven tumor growth. J. Clin. Invest 127, 2081-2090 (2017).

20. Gao, X. et al. MAP4K4 is a novel MAPK/ERK pathway regulator required for lung adenocarcinoma maintenance. Mol. Oncol. 11, 628-639 (2017).

21. Mouchemore, K. A. et al. Specific inhibition of PI3K p110delta inhibits CSF-1induced macrophage spreading and invasive capacity. FEBS J. 280, 5228-5236 (2013).

22. Jiang, $\mathrm{H}$. et al. Long noncoding RNA CRNDE stabilized by hnRNPUL2 accelerates cell proliferation and migration in colorectal carcinoma via activating Ras/MAPK signaling pathways. Cell Death Dis. 8, e2862 (2017).

23. Agajanian, M., Runa, F. \& Kelber, J. A. Identification of a PEAK1/ZEB1 signaling axis during TGF $\beta /$ fibronectin-induced EMT in breast cancer. Biochem Biophys. Res Commun. 465, 606-612 (2015). 
24. Li, L. et al. PLA2G16 promotes osteosarcoma metastasis and drug resistance via the MAPK pathway. Oncotarget 7, 18021-18035 (2016).

25. Yamasaki, T. et al. MicroRNA-218 inhibits cell migration and invasion in renal cell carcinoma through targeting caveolin-2 involved in focal adhesion pathway. J. Urol. 190, 1059-1068 (2013).

26. Chen, J. S. et al. Sonic hedgehog signaling pathway induces cell migration and invasion through focal adhesion kinase/AKT signaling-mediated activation of matrix metalloproteinase (MMP)-2 and MMP-9 in liver cancer. Carcinogenesis 34, 10-19 (2013).

27. Zhu, M. et al. Calcium-binding protein S100A14 induces differentiation and suppresses metastasis in gastric cancer. Cell Death Dis. 8, e2938 (2017).

28. Li, N. et al. MiR-106b and miR-93 regulate cell progression by suppression of PTEN via PI3K/Akt pathway in breast cancer. Cell Death Dis. 8, e2796 (2017).

29. Zhang, F. et al. EIF3D promotes gallbladder cancer development by stabilizing GRK2 kinase and activating PI3K-AKT signaling pathway. Cell Death Dis. 8, e2868 (2017)

30. Strnadel, J. et al. elF5A-PEAK1 signaling regulates YAP1/TAZ protein expression and pancreatic cancer cell growth. Cancer Res 77, 1997-2007 (2017).

31. Fujimura, K. et al. A hypusine-elF5A-PEAK1 switch regulates the pathogenesis of pancreatic cancer. Cancer Res 74, 6671-6681 (2014).

32. Ambros, V. \& Chen, $X$. The regulation of genes and genomes by small RNAs. Development 134, 1635-1641 (2007).

33. Lewis, B. P., Burge, C. B. \& Bartel, D. P. Conserved seed pairing, often flanked by adenosines, indicates that thousands of human genes are microRNA targets. Cell 120, 15-20 (2005).

34. Lu, J. et al. MicroRNA expression profiles classify human cancers. Nature $\mathbf{4 3 5}$ 834-838 (2005)
35. Hur, K. et al. MicroRNA-200c modulates epithelial-to-mesenchymal transition (EMT) in human colorectal cancer metastasis. Gut 62, 1315-1326 (2013).

36. Han, T. S. et al. MicroRNA-29c mediates initiation of gastric carcinogenesis by directly targeting ITGB1. Gut 64, 203-214 (2015).

37. Gasparini, P. et al. MicroRNA classifiers are powerful diagnostic/prognostic tools in ALK-, EGFR-, and KRAS-driven lung cancers. Proc. Natl. Acad. Sci. USA 112, 14924-14929 (2015)

38. Zhang, W. C. et al. Tumour-initiating cell-specific miR-1246 and miR-1290 expression converge to promote non-small cell lung cancer progression. Nat. Commun. 7, 11702 (2016).

39. Fabian, M. R., Sonenberg, N. \& Filipowicz, W. Regulation of mRNA translation and stability by microRNAs. Annu Rev. Biochem 79, 351-379 (2010).

40. Wang, X. F. et al. MiR-181d acts as a tumor suppressor in glioma by targeting K-ras and Bcl-2. J. Cancer Res Clin. Oncol. 138, 573-584 (2012).

41. Zhang, W. et al. miR-181d: a predictive glioblastoma biomarker that downregulates MGMT expression. Neuro Oncol. 14, 712-719 (2012).

42. Yang, F. et al. miR-181d/MALT1 regulatory axis attenuates mesenchyma phenotype through NF-KB pathways in glioblastoma. Cancer Lett. 396, 1-9 (2017).

43. Ikeda, Y., Tanji, E., Makino, N., Kawata, S. \& Furukawa, T. MicroRNAs associated with mitogen-activated protein kinase in human pancreatic cancer. Mol. Cancer Res 10, 259-269 (2012).

44. Guo, X. et al. miR-181d and c-myc-mediated inhibition of CRY2 and FBXL3 reprograms metabolism in colorectal cancer. Cell Death Dis. 8, e2958 (2017).

45. Wen, C. et al. Pseudolaric acid B induces mitotic arrest and apoptosis in both 5-fluorouracil-sensitive and -resistant colorectal cancer cells. Cancer Lett. 383, 295-308 (2016) 